\title{
Degradation studies of hydrophilic, partially degradable and bioactive cements (HDBCs) incorporating chemically modified starch
}

\author{
Ana C. Mendes • Luciano F. Boesel • \\ Rui L. Reis
}

Received: 26 July 2011/ Accepted: 11 January 2012

(C) Springer Science+Business Media, LLC 2012

\begin{abstract}
The degradation rate in Hydrophilic, Degradable and Bioactive Cements (HDBCs) containing starch/ cellulose acetate blends (SCA) is still low. In order to increase degradation, higher amounts of starch are required to exceed the percolation threshold. In this work, gelatinization, acetylation and methacrylation of corn starch were performed and assessed as candidates to replace SCA in HDBCs. Formulations containing methacrylated starch were prepared with different molar ratios of 2-hydroxyethyl methacrylate and methyl methacrylate in the liquid component and the amount of residual monomer released into water was evaluated. The concentration of reducing sugars, percentage of weight loss and morphologic analyses after degradation all confirmed increased degradation of HDBC with $\alpha$-amylase, with the appearance of pores and voids from enzymatic action. Methacrylated starch therefore is a better alternative to be used as the solid component of HDBC then SCA, since it leads to the formation of cements with a lower release of toxic monomers and more prone to hydrolytic degradation while keeping the other advantages of HDBCs.
\end{abstract}

\footnotetext{
A. C. Mendes $(\bowtie) \cdot$ L. F. Boesel · R. L. Reis

3B's Research Group, Biomaterials, Biodegradables and Biomimetics, Headquarters of the European Institute of Excellence on Tissue Engineering and Regenerative Medicine, University of Minho, AvePark, Taipas, 4806-909 Guimarães, Portugal e-mail: ana.mendes@dep.uminho.pt

A. C. Mendes · L. F. Boesel · R. L. Reis ICVS/3B's PT Government Associate Laboratory, Braga, Guimarães, Portugal
}

\section{Introduction}

Despite intense research aimed at improving the properties of conventional acrylic bone cements, they still present several problems. Some of the main problems are thermal $[1,2]$ and chemical necrosis $[1,3]$ of the bone attributed to the polymerization heat and release of residual monomers respectively, the weakness of cement interfaces with bone and prosthesis, shrinkage during polymerization and stress shielding of the bone due to improper transfer of load [1, 4]. The combination of such drawbacks leads to the most important problem found in these materials: the aseptic loosening of the implant on long-term with subsequent needs of a revision surgery.

Therefore, new formulations are clearly required with the aim of minimize some or all of these problems and consequently provide a stronger, stable and longer-lasting interface between bone and cement. One alternative could be the preparation of partially degradable bone cements that would be able to promote the growth of bone on the surface as well as in the interior of the cement. Following this assumption, the gradual degradation of the degradable component would generate pores and voids that could be filled by bone, growing due to the bioactive character provided by the bioactive fillers [5].

Hydrophilic partially degradable and bioactive cements (HDBCs) were developed as novel class of acrylic-based bone cements [1], containing a hydrogel forming monomer (2-hydroxyethyl methacrylate (HEMA)) in liquid component, replacing partially the common methyl methacrylate (MMA) in order to improve mechanical [6, 7] and swelling $[6,8,9]$ properties of the system. In spite of the change in composition of such cements, the main characteristics of this cements, namely the fast polymerization rate, injectability and higher mechanical properties are maintained [1]. 
In addition, the incorporation of HEMA in these formulations could promote good compatibility [10] with body fluids and tissues allowing the controlled release of bioactive agents and enhance bioactivity [11].

These cements also incorporate starch based materials that can degrade into the body by hydrolysis of $\alpha-1-4$-glycosidic linkages of starch to oligosaccharides, maltose, maltotriose and predominantly glucose [12-14]. This degradation is catalysed by $\alpha$-amylase, enzyme present at low concentrations in human serum. The common starch-based material used in HDBCs until now was a blend of starch with cellulose acetate (SCA) [1] which has already been studied for a range of biomedical applications such as scaffolds [15] for tissue engineering, systems for drug delivery [16] and hydrogels [17] among others. SCA degrades under the presence of $\alpha$-amylase [14], however, when grafted with other material such as $30-45 \%$ of acrylic polymers in the cement formulation, the access of the enzyme to the starch is hindered. To change this scenario, the percentage of starch in the material should be higher than the percolation threshold $\left(\mathrm{p}_{\mathrm{c}}\right)$, that is the minimum concentration that creates full connectivity from the bottom to the top of surface [18]. In this case the degradation of starch would create a connected network of pores and voids that would allow the transport of the enzyme from the surface to the interior of material. In addition, an increase in surface roughness is expected as a result of degradation which could induce a stronger interface with bone.

Boesel et al. [19] concluded that the volume fraction of starch provided by SCA in HDBC was not sufficient to create percolation and for this reason only a small degradation was achieved in these formulations.

In this article we assessed the suitability to incorporate modified corn starch as replacement to the SCA used in original HDBC formulations, in order to reach percolation threshold. Gelatinized, acetylated as well as methacrylated corn starch were prepared and characterized and their application in HDBC was analyzed in terms of weight loss and morphology. Formulations prepared with methacrylated starch were further characterized in terms of degradation behaviour and release of residual monomers.

\section{Materials and methods}

\subsection{Materials}

The starch provided by Copam (Companhia Portuguesa de Amidos SA, Portugal) was modified with acetic anhydride (Merck, Germany) and methacrylic anhydride (Aldrich, Germany). Corn starch/cellulose acetate (SCA) blend 50/50 $\mathrm{wt} \%$ was provided by Novamont, Italy. These materials were employed in the solid phase of bone cements. In the liquid component, methylmethacrylate (MMA, Merck, Germany) as well as 2-hydroxyethyl methacrylate (HEMA, Fluka, Germany) were used as received. Benzoyl Peroxide (BPO, Merck, Germany) was used as polymerization initiator with previous purification by fractional recrystallization from ethanol and subsequently vacuum dried. The activator was dimethyl-p-toluidine (DMT, Aldrich, Germany).

\subsection{Structural modifications and characterization} in native corn starch

\subsubsection{Gelatinization of corn starch}

25 wt $\%$ of corn starch was suspended in water under magnetic stirring and heated $\left(80^{\circ} \mathrm{C}\right)$ in order to gelatinize the starch. The gelatinized corn starch was dried at $60^{\circ} \mathrm{C}$ over a period of $12 \mathrm{~h}$.

\subsubsection{Acetylation of corn starch}

Native corn starch was dried in oven at $50^{\circ} \mathrm{C}$ over a period of $24 \mathrm{~h} .30 \mathrm{~g}$ of dried starch were mixed with acetic anhydride at weight ratio 1:2 under magnetic stirring. After 5 min of stirring, $50 \% \mathrm{NaOH}$ was added at a ratio of $0.22 \mathrm{~g} / \mathrm{g}$ starch. Temperature was increased to $123^{\circ} \mathrm{C}$ within $15 \mathrm{~min}$ and kept for $2.5 \mathrm{~h}$. Excess of cold water was added to finish reaction and wash the reaction products.

\subsubsection{Methacrylation of corn starch}

This step was performed following the method reported by Caldwell [20]: $40 \mathrm{wt} \%$ of corn starch were suspended in water at room temperature and $\mathrm{pH}$ raised to approximately 9 with $\mathrm{NaOH} 3 \%$. Under constant magnetic stirring, methacrylic anhydride was added slowly at weight ratio 1:10 relatively to starch, maintaining $\mathrm{pH}$ between 8 and 9 by additions of $\mathrm{NaOH} 3 \%$. Agitation was continued for $1 \mathrm{~h}$ and then $\mathrm{pH}$ decreased until 6.5-7. The starch was washed with excess of cold water and dried.

\subsubsection{Structural analyses of modified starch}

2.2.4.1 FTIR analyses The information on structural modification on modified starches was collected by infrared spectra, with an IR Prestige-21(SHIMADZU, Japan) spectrophotometer, using $\mathrm{KBr}$ pellets.

2.2.4.2 Determination of degree of substitution(DS) $\quad 0.5 \mathrm{~g}$ of modified corn starch were placed in a conical flask and $50 \mathrm{~mL}$ of distilled water were added under magnetic stirring. The $\mathrm{pH}$ was adjusted to 7 with $\mathrm{NaHCO}_{3} 0.1 \mathrm{M}$. After that, $25 \mathrm{~mL}$ of $0.5 \mathrm{~N}$ of $\mathrm{NaOH}$ were added and the sample was heated until the solution became transparent. Excess of $\mathrm{NaOH}$ was titrated with $\mathrm{HCl} 0.02 \mathrm{~N}$ back to $\mathrm{pH}$ 7. Triplicate 
preparations were performed to obtain a mean value. DS was calculated as:

$\mathrm{DS}=\frac{M_{g} \times\left(N_{\mathrm{NaOH}} \times V_{\mathrm{NaOH}}-N_{\mathrm{HCl}} \times V_{\mathrm{HCl}}\right)}{1.00 \times m_{s} \times M_{\mathrm{An}} \times\left(N_{\mathrm{NaOH}} \times V_{\mathrm{NaOH}}-N_{\mathrm{HCl}} \times V_{\mathrm{HCl}}\right)}$

Eq. 1 was employed for methacrylated and for acetylated corn starch. $N_{\mathrm{NaOH}}$ and $V_{\mathrm{NaOH}}$ are the normality and the volume of $\mathrm{NaOH}$, respectively. $N_{\mathrm{HCl}}$ and $V_{\mathrm{HCl}}$ are the normality and the volume of $\mathrm{HCl}$, respectively used to back titrate. $M_{\mathrm{g}}$ is the molecular weight of glucose skeleton (162) on which reaction took place; $m_{\mathrm{s}}$ is the mass of modified starch $(0.5 \mathrm{~g})$ used for quantification; $M_{\mathrm{An}}$ is the molecular weight of the acetyl group coming from acetic anhydride (42) and methacrylic group (68) provided from methacrylic anhydride.

\subsection{Preparation and selection of formulations and solutions}

Cement specimens were prepared by adding the solid component to the liquid component. The solid was constituted by the modified starches having particles size $<250 \mu \mathrm{m}$. In one formulation, instead of modified starch, a blend of corn starch/cellulose acetate 50/50 wt\% (SCA) was used for comparison. BPO was used as the polymerization initiator at a molar concentration of 0.016 with respect to the monomer amount (that is $1.1 \mathrm{wt} \%$ of the solid). The liquid was constituted by a mixture of HEMA and MMA at molar ratios between of 1/4 and 4/1 (HEMA/MMA). DMT was used as the activator of the initiator at a molar concentration of 0.37 with respect to the BPO amount (i.e., $0.6 \mathrm{wt} \%$ of the liquid). The solid/liquid ratio employed was 60/40.

Preparation of specimens for subsequent tests was carried out following the traditional method. The activator DMT was dissolved in the liquid phase. Initiator of free radical polymerization reaction was added to the solid phase. Both phases were mixed and stirred by hand until the mixture became paste-like with a high viscosity; the mass was then placed into a poly(tetrafluoroethylene) (PTFE) mold to allow it to cure as bar-shaped specimens with $\left(50 \times 5 \times 1.50 \mathrm{~mm}^{3}\right)$ of dimension.

\subsubsection{Percentage of weight loss (\% WL)}

In order to infer about the cohesion of the prepared formulations, the bar-shaped specimens triplicates were immersed in distilled water at a ratio of 1:40 (specimen mass: solution volume) and incubated at $37^{\circ} \mathrm{C}$ under dynamic conditions over a period of 7 days. After this period, the specimens were taken out of the solution, rinsed with distilled water, blotted on filter paper to remove surface solution/water, and immediately weighed. They were then dried until constant weight in order to determine the percentages of weight loss following Eq. 2

$W L_{t}=\left(\frac{m_{\mathrm{f}}-m_{0}}{m_{0}}\right) \times 100$

$m_{\mathrm{f}}$ is the final mass after immersion and drying and $m_{0}$, the initial mass prior to immersion.

\subsubsection{Scanning electron microscopy (SEM)}

The morphology of the specimens before and after incubation in the water bath at $37^{\circ} \mathrm{C}$ was analyzed with a Leica Cambridge S360 (Leica Cambridge Ltd., Cambridge, UK) SEM. Before observation, specimens were dried at room temperature and gold-coated by ion sputtering (Sputter Coater SC502, Fisons Instruments, UK).

\subsubsection{Residual monomer released into water by ${ }^{1} H$ NMR}

Formulations with different ratios HEMA-MMA, containing methacrylated starch in the solid component were prepared in order to quantify the percentage of residual monomer released into water. One formulation with SCA was prepared for comparison. Two specimens of each type were grounded immediately after preparation and dissolved in deuterated water at a ratio of 1:6 (specimen mass: solution volume) in order to extract residual monomer. The samples were incubated at $37^{\circ}$ under dynamic conditions during $48 \mathrm{~h}$. At the end of incubation time, samples were filtered to remove solid component, and the liquid was kept for further ${ }^{1} \mathrm{H}$ NMR analyses. Dimethylformamide (DMF) was added as an internal reference. All samples and DMF amounts were weighed on addition to the NMR tube. Fully relaxed ${ }^{1} \mathrm{H}$ NMR spectra were acquired in a Varian Unity Plus $300 \mathrm{MHz}$ spectrometer and the areas of the protons signals of MMA (6.12-6.10 ppm) and HEMA (6.18-6.15 ppm) relative to the DMF methyl signals (2.97-2.883 ppm) were quantified to give the quantity of residual monomer released to distilled water.

2.4 Characterization of HDBCs with methacrylated starch in terms of curing and degradation parameters

\subsubsection{Variation of the temperature with time (curing parameters)}

Dough time $\left(t_{\mathrm{d}}\right)$, setting time $\left(t_{\mathrm{s}}\right)$, and maximum polymerization temperature $\left(T_{\max }\right)$ were measured with the use of a Teflon mold, and a thermocouple connected to a digital display. The powder and liquid components of bone cements were mixed and packed into the mold. The thermocouple was located in the center of the interface between the curing mass (the cement) and the PTFE mould. Setting time was 
considered as the time at which the temperature of the mass was the sum of the test temperature $\left(25^{\circ} \mathrm{C}\right)$ and maximum temperature $(T \max )$ divided by two. This procedure was based on the international standard specification (ISO 5833) [21].

\subsubsection{Degradation tests in formulation with methacrylated starch}

The degradation behaviour of the prepared bone cement formulations was studied over a period of 4 weeks. Barshaped specimens were immersed in phosphate buffered saline solution (PBS, 0.01 M, pH 7.4) and PBS supplemmented with $150.5 \mathrm{U} / \mathrm{L}$ of convencional $\alpha$-amylase, from Aspergillus oryzae, at a ratio of 1:40 (specimen mass:solution volume) and incubated at $37^{\circ} \mathrm{C}$ under static conditions. The specimens were removed at specified intervals, rinsed with distilled water, blotted on filter paper to remove surface solution/water, and immediately weighed. They were then dried to constant weight in order to determine the percentages of weight loss and water uptake. The degradation solution was frozen for later analysis (determination of reducing sugars in solution). The percentage of weight loss (\%WL) was calculated following Eq. 2 and water uptake (WU) using the following equation

$\mathrm{WU}_{t}=\left(\frac{m_{t}-m_{\mathrm{f}}}{m_{\mathrm{f}}}\right) \times 100$,

where $m_{\mathrm{t}}$ is the wet mass of the specimen at time $t$ (days) and $m_{\mathrm{f}}$ is the final mass after immersion and drying.

The concentration of reducing sugars of all degradation solutions was determined using the dinitrosalicylic acid (DNS) method. For this purpose, DNS reagent was added and mixed with standard glucose concentration, control samples (distilled water, buffer solution) and samples of unknown concentration in the same proportion (sample/DNS). Samples were placed in a water bath $\left(100^{\circ} \mathrm{C}\right)$ and after boiled, they were cooled and diluted 10 times with distilled water. The DNS procedure is based on the reduction of the 3,5-dinitrosalicylic acid to 3-amino-5-nitrosalicylic acid under alkaline conditions, being the carbonyl group of sugars oxidized producing carboxylic groups. During this reaction there is the development of a colored complex whose intensity can be measured at $540 \mathrm{~nm}$. Quantification of reducing sugars in the degradation solutions was performed in a SpectraMax 340PC microplate spectrophotometer (Molecular Devices Corp., Sunnyvale) and using a calibration curve of standard glucose concentrations. The morphology of the specimens after degradation was analyzed by SEM as described previously.

\subsection{Statistical analysis}

Results of percentage of weight loss (WL), curing parameters, reducing sugars, and percentage of water uptake, are expressed as a mean \pm standard deviation with $n=3$ for each group. Statistical significance was determined using unpaired Student's $t$-test multiple comparison procedure; differences were considered significant if $p<0.05$.

\section{Results and discussion}

\subsection{Structural analyses of modified starch}

FTIR analyses (Fig. 1) show the characteristics absorption bands of native corn starch occurring at $3480 \mathrm{~cm}^{-1}(-\mathrm{OH}$ stretch from glycosidic ring); $2990 \mathrm{~cm}^{-1}$ (C-H stretch), $1630 \mathrm{~cm}^{-1}$ (-OH bending from water); $1350 \mathrm{~cm}^{-1}$ (-OH bending); $1000 \mathrm{~cm}^{-1}$ (C-O-C stretching from anhydroglucose ring). As expected, the gelatinization did not have
Fig. 1 Infrared spectrum of gelatinizated, native, methacrylated and acetylated corn starch evidencing the structural modification on native corn starch

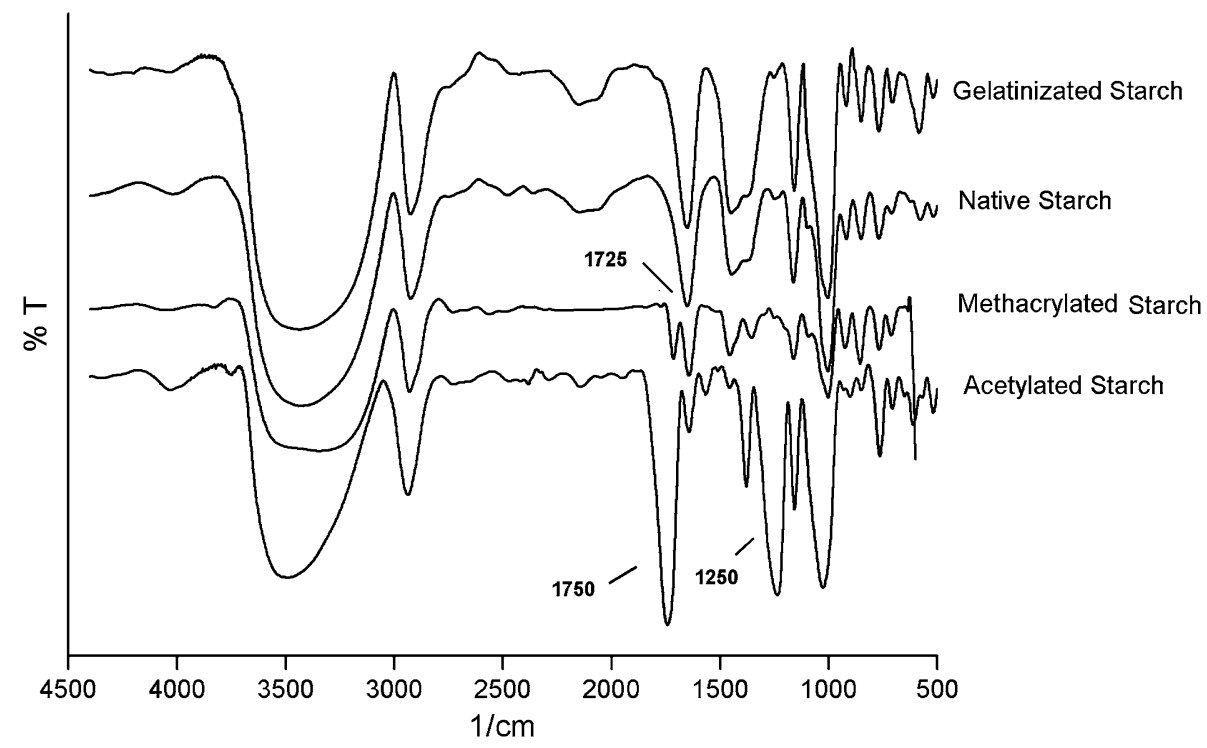


any effect on functional structure of starch and for this reason the two FTIR spectra of Native and Gelatinized starch are quite similar. In the spectrum of acetylated starch, the appearing of three strong bands corresponding to the acetate group at $1750 \mathrm{~cm}^{-1}(\mathrm{C}=\mathrm{O}$ stretching band), $1375 \mathrm{~cm}^{-1}\left(\mathrm{C}-\mathrm{CH}_{3}\right.$ deformation band) and $1250 \mathrm{~cm}^{-1}$ (C-O stretching band) and the decrease of $-\mathrm{OH}$ bending from native corn starch around $1350 \mathrm{~cm}^{-1}$, confirm the success of this reaction by the method employed. The FTIR spectrum of methacrylated starch shows that intensity of absorption bands at 1651 and $1350 \mathrm{~cm}^{-1}$, attributed to the -OH bending in the corn starch decreased after the reaction with methacrylic anhydride and the peaks of ester carbonyl bond appeared at $1725-1730 \mathrm{~cm}^{-1}$. Therefore, it might be concluded that such structural modifications on native corn starch were successful.

\subsubsection{Degree of substitution (DS)}

The DS for a starch derivative is defined as moles of substituents of hydroxyl groups per anhydroglucose structural unit of the starch polymer and affects the functional properties of starch, including enzymatic attack for degradation [22]. The value for DS varies from 0 to 3 and we found DS of 1.76 for methacrylic starch and DS 1.2 for acetylated starch.

\subsection{Preparation and selection of formulations}

\subsubsection{Percentage of weight loss (\% WL)}

Gelatinization of starch is the disruption of molecular order within granules [23]. Extensive heating in excess water

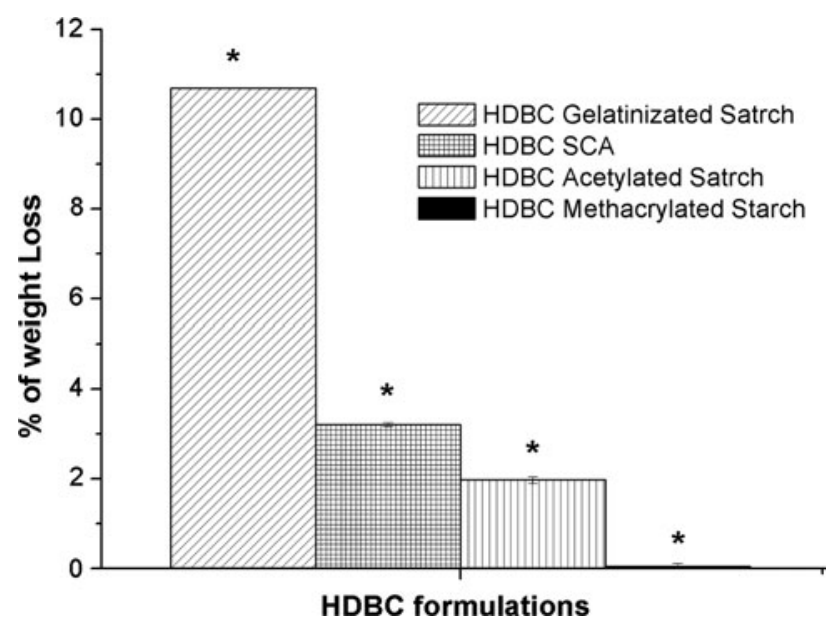

Fig. 2 Percentage of weight loss for different HDBC formulations after 1 week immersed in shaker water bath at $37^{\circ} \mathrm{C}$. Results are expressed as mean \pm standard deviation with $n=3$. *Indicates a significant difference $(p<0.05)$ between all formulations causes swelling and then rupture of starch granules, loss of cristalinity and release of soluble material from the granules [24]. Generally the amorphous parts of granules are substituted more easily than the crystalline areas [25]. Native corn starch did not react with acrylic monomers and for this reason it was gelatinized aiming at enhancing chemical reactivity of inert starch granules on grafting as well as improving enzymatic hydrolysis.

In spite of this increase in reactivity of native corn starch by gelatinization, it was not sufficient to induce the grafting reaction between acrylic monomers and starch in HDBC formulations. After 1 week of immersion in water at $37^{\circ} \mathrm{C}$ these HDBCs containing gelatinizated starch lost $10.7 \%$ of their total mass (Fig. 2), the highest $(p<0.05)$ value of weight loss $(\%)$ found in the four HDBCs formulations analyzed. Figure 3 evince that the grafting efficiency for gelatinizated starch is low after immersion in water. The surface of this cement appears disrupted, due to the lack of cohesion between solid and liquid components, caused by the lack of interaction between modified starch and acrylic monomers. As refered by Hebeish et al. [26], in the grafting of corn starch with methyl methacrylate, side chains resulting at a frequency of one glucose unit per 230-300 glucose units were observed, meaning that the yield of this kind of reaction is too low. In fact the only interaction between solid and liquid components could be attributed to hydrogen bond interactions between hydroxyl groups presented in gelatinizated corn starch and HEMA.

In order to promote starch grafting several modifications such as etherification, acetylation, or esterification with vinyl monomers are required to introduce a reaction site for the further formation of a copolymeric chain [27]. HDBC prepared with SCA presents a lost of mass of about $3.2 \%$ (Fig. 2). Accordingly, in SCA only CA presents reactive sites for grafting reaction while the starch (which amounts to $50 \%$ of the blend) is prone to be leached. This fact (together with the presence of soluble oligomers and plasticizers) may explain the higher weight loss for formulations containing SCA as the solid component in comparison with acetylated and methacrylated ones.

Comparing now methacrylated and acetylated starch the percentage of weight loss (Fig. 2) and the morphological analysis of surface after 1 week immersed in water (Fig. 3), suggests that the grafting for methacrylated starch works better than for acetylated starch. HDBC prepared with acetylated starch presents a lost of mass of about $2 \%$ while HDBC with methacrylated starch had no relevant weight loss after 1 week of immersion in water (Fig. 2), being this difference statistically significant $(p<0.05)$. We suppose that this fact may be related with the similarity in chemical structure of methacrylated with acrylic monomers. When a grafting reaction takes place polymer chains wrap the carbohydrate's backbone, 
Fig. 3 SEM micrographs of the surface of HDBCs prepared with the methacrylated, acetylated, and gelatinizated starch and SCA at day 0 and after 7 days of immersion in a shaker water bath
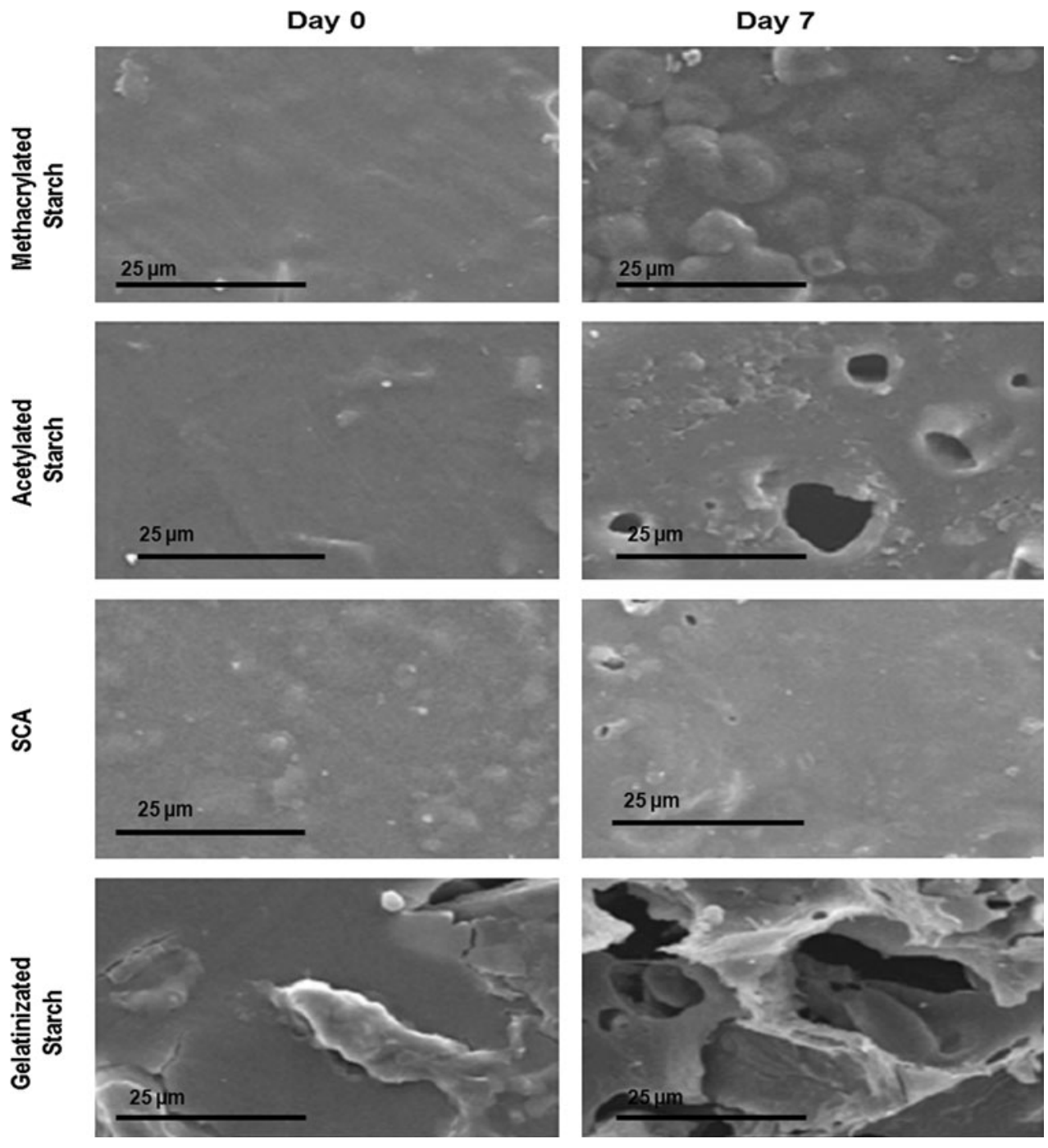

increasing the free volume of the total product and consequently decreasing its density [28]. We assumed that methacrylated starch induces the formation of polymers with lower density and a decrease of the viscosity in the system that allow better diffusion of acrylic monomers into the reacting system.

The majority of this measured weight loss can be attributed to the starch granules that did not react with acrylic monomers. On top of that, the release of residual monomer and the leaching of low molecular weight molecules, which are soluble in water and may be released after water absorption, also contribute to these observations.

The morphology of the surface of starch has been well studied by several authors. SEM micrographs usually show that native starch structure is formed by oval granules. The image analysis (Fig. 3) reveals the formation of pores with little shapes and sizes as a result of sorption of water after 7 days of immersion, as well as different interactions between acrylic monomers and each modified starch. After the period of immersion in water bath the oval granules of methacrylated starch becomes salient, confirming that after the leaching out of the structure of starch is partially kept after chemical modification (Fig. 3).

Once the HDBCs prepared with methacrylated starch have demonstrated the major potential for the application, the methacrylated starch was selected for the subsequent cements studies.

\subsubsection{Residual monomer released into water by ${ }^{1} \mathrm{H} N M R$ of HDBC prepared with methacrylated starch}

Different formulations containing methacrylated starch were prepared with different ratios MMA/HEMA in order to analyse the residual monomer released into water by ${ }^{1} \mathrm{H}$ NMR. The amounts of MMA as well as HEMA monomer released were estimated by taking into consideration the resonance signal of vinylic protons (5.8-6.2 ppm) as shown in Fig. 4 . This procedure also allowed us to compare the relative reactivity of each acrylic monomeric unity in the curing process of the bone cement. 
Fig. $4{ }^{1} \mathrm{H}$ NMR of HDBC with methacrylated starch in which HEMA $=1 / 2$ MMA, evidencing the vynilic protons of acrylic monomers

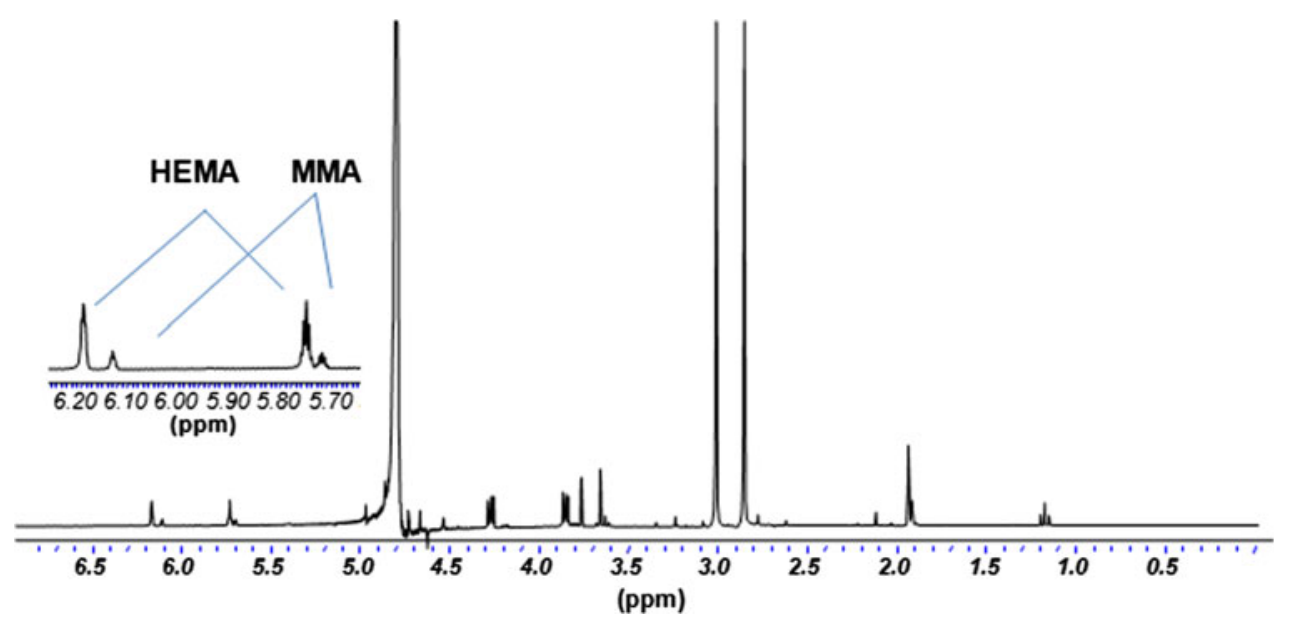

The percentage of residual monomer was found to increase with the increase of the MMA content with respect to HEMA from 0.25 to 4 (Table 1). In all samples the percentage of residual HEMA was higher than the percentage of residual MMA meaning that MMA seems to be more reactive in this system in terms of grafting with starch than HEMA. Generally radical polymerization in acrylic bone cements does not proceed to completion [29] due to the decrease of mobility of monomers by the increase in viscosity at high conversion rates.

Therefore, it can be hypothesized that formulations prepared with methacrylated starch are less toxic than those ones performed with SCA. The formulation with lower release of acrylic monomer is the one with higher content of HEMA. Nevertheless due to the hydrophilicity of this monomer, higher water sorption as well as a decrease in mechanical properties should be expected and for this reason the selected formulation for further analyses was the one with HEMA $=1 / 2$ MMA.

3.3 Characterization of HDBC with methacrylated starch in terms of curing and degradation parameters

\subsubsection{The variation of the temperature with time (curing parameters)}

As expected, the curing parameters are strongly affected by the composition of the cement. Biomet Plus is a

Table 1 Amount of released residual monomer quantified by ${ }^{1} \mathrm{H}$ NMR

\begin{tabular}{lcl}
\hline Sample (monomers ratio) & \% HEMA released & $\%$ MMA released \\
\hline MMA $=$ 4HEMA & $1.26-1.29$ & $0.11-0.14$ \\
$\mathrm{MMA}=$ 2HEMA & $0.54-0.86$ & $0.07-0.11$ \\
$\mathrm{MMA}=$ 2HEMA (SCA) & $1.24-1.36$ & $0.20-0.31$ \\
$\mathrm{MMA}=1 / 4 \mathrm{HEMA}$ & $0.3-0.38$ & Non detected \\
\hline
\end{tabular}

commercial bone cement containing PMMA beads in the solid component and MMA monomer in the liquid. The HDBC is constituted from methacrylated starch in the solid component and by MMA and HEMA monomers in the liquid. Due to the presence of the hydrophilic monomer in HDBC, a higher enthalpy of polymerization is expected resulting in an increase of maximum polymerization temperature $\left(T_{\max }\right)$. It can be observed in Fig. 5 that $T_{\max }$ for HDBC is $(66 \pm 1.18){ }^{\circ} \mathrm{C}$ while for Biomet Plus $T_{\max }$ it is $(63 \pm 0.57){ }^{\circ} \mathrm{C}$.

The difference in $T_{\max }$ from HDBC to the commercial one is not statistically significant $(p>0.05)$ suggesting that the shrinkage effect and thermal necrosis should be minimized relatively to the other HDBC's formulations with higher polymerization temperatures. The calculated setting time was around $3 \mathrm{~min}$, a value close to those found for other HDBC's formulations [1]. This value is shorter than that usually found for commercial bone cements or that required from the standards $\left(t_{\mathrm{s}}>5 \mathrm{~min}\right)$. The short time originates from the use of a more reactive acrylic monomer (HEMA) and the fact that the monomers interact with the surface of the filler, without diffusion into the particles as

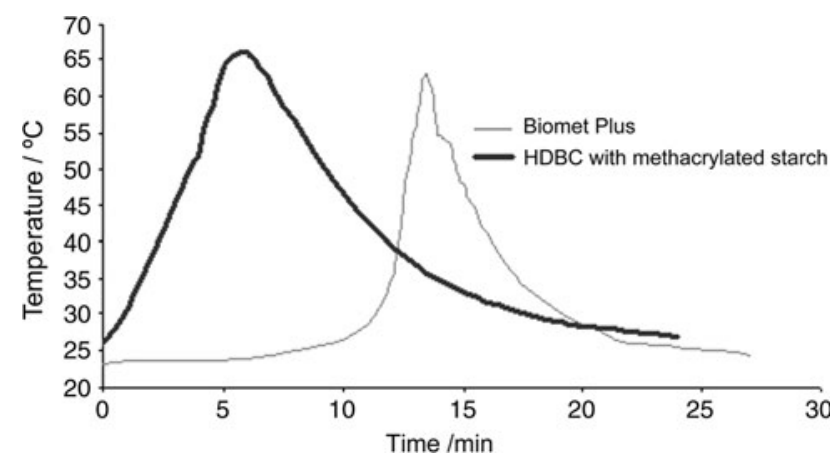

Fig. 5 Curing curves of HDBC with methacrylated starch and comparison with commercial Biomet Plus acrylic bone cement. The difference in the Tmax for both materials is not significant statistically $(p>0.05)$ 
in commercial cements. Without such diffusion, polymerization is not retarded and the monomers-polymer reach the gel point earlier, leading also to a shorter setting time. It should be emphasized that we did not add any extra polymerization inhibitor-retarder to the monomers (they were used as received). By doing that, we may extend setting time to values in agreement with the standards.

\subsubsection{Degradation tests in formulation with methacrylated starch}

After 4 weeks, higher concentration of reducing sugars was found in samples immersed in PBS supplemented with $\alpha$-amylase $(0.42 \mathrm{mg} / \mathrm{mL})$ than in PBS without enzyme $(0.01 \mathrm{mg} / \mathrm{mL}$, Fig. 6), confirming that degradation of methacrylated starch is promoted by $\alpha$-amylase. In PBS, only after the 2nd week a small amount of reducing sugars was detected. The differences between the concentration of

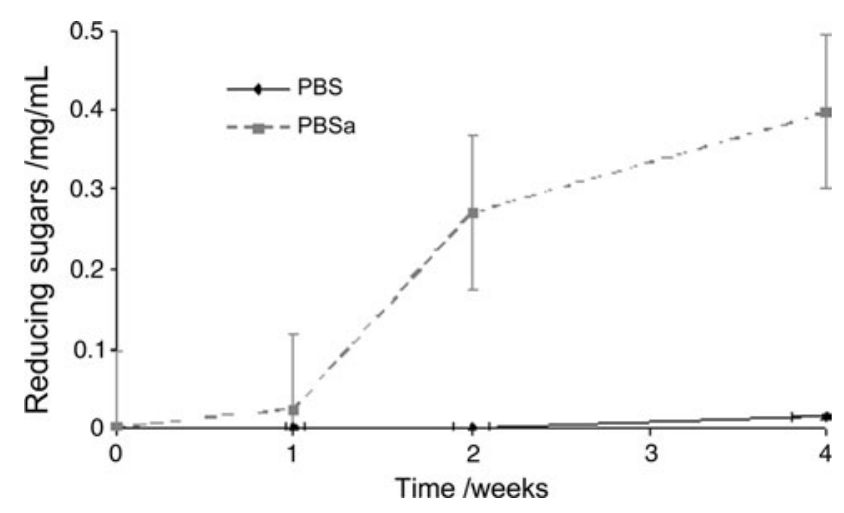

Fig. 6 Degradation profile of HDBC with methacrylated starch at $37^{\circ} \mathrm{C}$ in PBS solution (pH 7.4) with (PBSa) and without (PBS) $\alpha$-amylase (150 U/L) in terms of concentration of reducing sugars in solution. Significant differences $(p<0.05)$ between samples immersed in PBS and PBSa were found for all time points

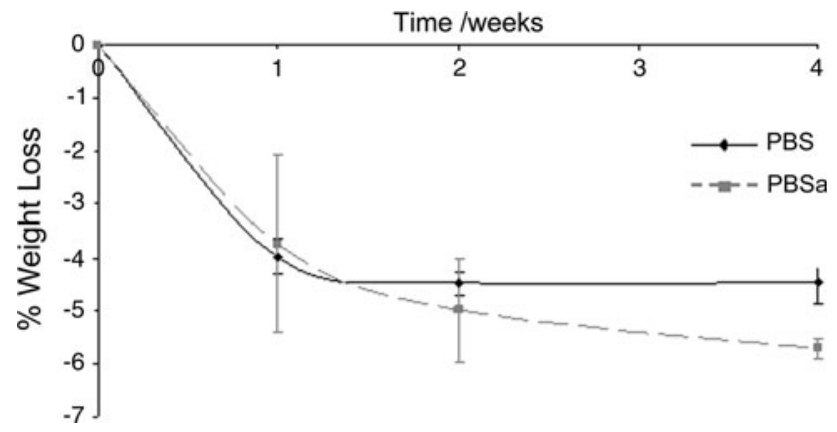

Fig. 7 Degradation profile of HDBC with methacrylated starch at $37^{\circ} \mathrm{C}$ in PBS solution (pH 7.4) with (PBSa) and without (PBS) $\alpha$-amylase $(150 \mathrm{U} / \mathrm{L})$ in terms of weight loss in solution. Significant differences $(p<0.05)$ between samples immersed in PBS and PBSa were found only at 4 weeks reducing sugars for samples in PBS and PBSa were found to be statistically significant $(p<0.05)$ at all time points.

An increase in percentage of weight loss is observed in both situations, PBS and PBSa until the 1st week (Fig. 7). However, after the 1st week, samples immersed in PBS kept their weight while samples in PBSa continued to lose weight being the difference for the last time point statistically significant $(p<0.05)$. It might be concluded that weight loss until the 1st week was not just due to enzymatic degradation but also to the leaching of low molecular weight molecules as well plasticizers and non-polymerized monomers or oligomers, which are soluble in water and may be released after water uptake. The soluble low molecular weight sugars released into solution become more accessible for enzymatic attack and otherwise the empty spaces previously occupied by these released molecules allow more easy access of the enzyme to the polymeric matrix.

Until the first week a noticeable increase in water absorption (Fig. 8) in both situations PBS and PBSa is confirmed $(p<0.05)$. Nevertheless after the first week of study, due to the water absorption, the initiation of enzymatic attack started to be more efficient in terms of concentration of reducing sugars and weight loss (Figs. 6, 7) creating more points for water absorption as well as accelerating the degradation process. Higher water absorption accelerates the degradation process [30]. The degradation can be related with an increase in permeability of the materials caused by removal of starch fraction from the sample, as a result of enzymatic hydrolysis [14]. One should expect the appearance of pores and voids in the volume previously occupied by the starch and in fact SEM micrographs of these HDBC surfaces showed that samples immersed in PBSa present pores and voids, whose diameter and depth seems to increase with time (Fig. 9). The pore size seems to have round shape (Fig. 9) characteristic from the particles of methacrylated starch as showed in Fig. 3.

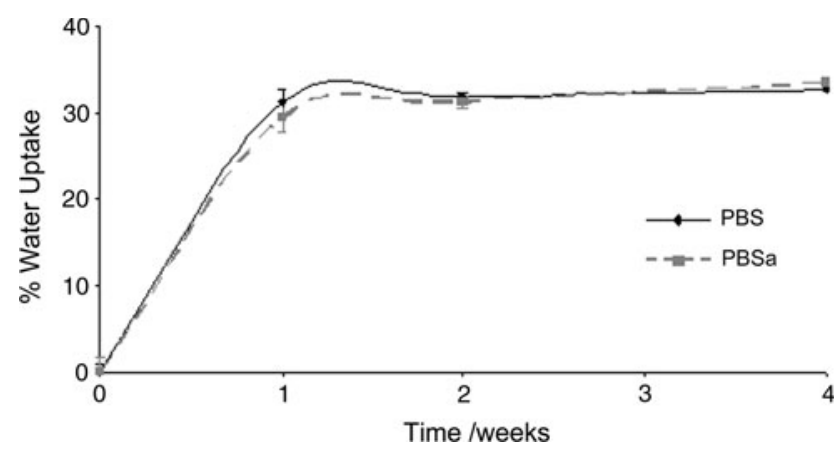

Fig. 8 Water uptake of $\mathrm{HDBC}$ with methacrylated starch at $37^{\circ} \mathrm{C}$ in PBS solution (pH 7.4) with (PBSa) or without (PBS) $\alpha$-amylase (150 U/L). Significant differences $(p<0.05)$ between samples immersed in PBS and PBSa were found for the first time point (1 week) 

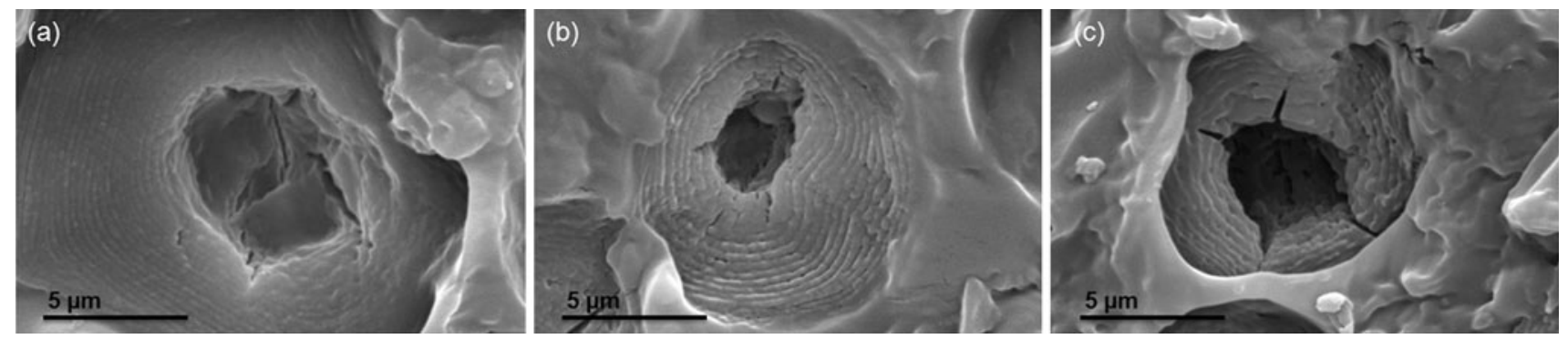

Fig. 9 SEM micrographs of surfaces of specimens (HDBCs containing methacrylated starch) after immersion in PBSa: 1 week (a), 2 weeks (b) and 3 weeks (c) demonstrating the ability of this matrix to be degraded enzymatically

This could confirm that, in general, these pores were formed by means of degradation of the particulate filler, the starch or in this specific case methacrylated starch. Moreover, it helps to confirm that the methacrylated starch structure was partially kept after reaction with acrylic monomers.

However, some additional tests with longer degradation time need to be performed in order to evaluate the possibility of forming an interconnected network of pores. This interconnectivity plays an important role in the successful application of HDBCs, since in vivo, with higher degree of interconnectivity, bone will be able to grow inside these pores, as discussed elsewhere [19].

\section{Conclusions}

This study revealed that different modifications in starch promote different behaviours in terms of its reactivity on grafting with acrylic monomers being methacrylated starch the most promising one. The use of methacrylated starch in our current formulation comparatively to SCA revealed more efficiency in terms of grafting and consequently leads to the formation of cements with a lower release of toxic monomers. Moreover the methacrylated starch allowed us to solve the problem of low amount of starch to achieve the percolation threshold and consequently enhance biodegradable properties in HDBC, making it a better alternative for the solid component of HDBC them SCA. Therefore, full connectivity between pores and voids from degradation were not detected for 4 weeks of study, being required more time for analyses. This interconnectivity plays an important role in the successful application of HDBCs, since in vivo, with higher degree of interconnectivity, bone will be able to grow inside these pores.

To improve better performance of HDBCs, apart from degradation, bioactivity should be employed for further promotion of bone growth at later stages by cellular adhesion with consequently improvement of the interface with bone.
Acknowledgments The authors acknowledge to Foundation for Science and Technology (FCT), who supported this study through funds from project Concept2Cement (POCTI/CTM/60735/2004).

\section{References}

1. Boesel LF, Reis RL. A review on the polymer properties of hydrophilic, partially degradable and bioactive acrylic cements (HDBC). Prog Polym Sci. 2008;33(2):180-90.

2. Ormsby R, McNally $\mathrm{T}$, Mitchell C, Halley $\mathrm{P}$, Martin D, Nicholson T, Dunne N. Effect of MWCNT addition on the thermal and rheological properties of polymethyl methacrylate bone cement. Carbon. 2011;49(9):2893-904.

3. Bettencourt AF, Neves CB, de Almeida MS, Pinheiro LM, Oliveira SA, Lopes LP, Castro MF. Biodegradation of acrylic based resins: a review. Dental Mater. 2010;26(5):171-80.

4. Gladius L. Alternative acrylic bone cement formulations for cemented arthroplasties: present status, key issues, and future prospects. J Biomed Mater Res B Appl Biomater. 2008;84B(2):301-19.

5. Boesel LF, Cachinho SCP, Fernandes MHV, Reis RL. The in vitro bioactivity of two novel hydrophilic, partially degradable bone cements. Acta Biomater. 2007;3(2):175-82.

6. Boesel LF, Reis RL. The effect of water uptake on the behaviour of hydrophilic cements in confined environments. Biomaterials. 2006;27(33):5627-33.

7. Boesel LF, Mano JF, Reis RL. Optimization of the formulation and mechanical properties of starch based partially degradable bone cements. J Mater Sci: Mater Med. 2004;15(1):73-83.

8. Boesel LF, Fernandes MHV, Reis RL. The behavior of novel hydrophilic composite bone cements in simulated body fluids. J Biomed Mater Res B Appl Biomater. 2004;70B(2):368-77.

9. Boesel L, Reis R. Hydrophilic matrices to be used as bioactive and degradable bone cements. J Mater Sci: Mater Med. 2004; 15(4):503-6.

10. Omidian H, Park K, Kandalam U, Rocca JG. Swelling and mechanical properties of modified HEMA-based superporous hydrogels. J Bioact Compat Polymer. 2010;25(5):483-97.

11. Koleganova VA, Bernier SM, Dixon SJ, Rizkalla AS. Bioactive glass/polymer composite materials with mechanical properties matching those of cortical bone. J Biomed Matter Res A. 2006; 77(3):572-9.

12. Rosa DS, Lopes DR, Calil MR. Thermal properties and enzymatic degradation of blends of poly([epsilon]-caprolactone) with starches. Polym Testing. 2005;24(6):756-61.

13. AlbertaAraújo M, Cunha AM, Mota M. Enzymatic degradation of starch-based thermoplastic compounds used in protheses identification of the degradation products in solution. Biomaterials. 2004;25(13):2687-93. 
14. Azevedo HS, Gama FM, Reis RL. In vitro assessment of the enzymatic degradation of several starch based biomaterials. Biomacromolecules. 2003;4(6):1703-12.

15. Pashkuleva I, Marques AP, Vaz F, Reis RL. Surface modification of starch based blends using potassium permanganate-nitric acid system and its effect on the adhesion and proliferation of osteoblast-like cells. J Mater Sci: Mater Med. 2005;16(1):81-92.

16. Silva GA, Costa FJ, Neves NM, Coutinho OP, Dias ACP, Reis RL. Entrapment ability and release profile of corticosteroids from starch-based microparticles. J Biomed Mat Res A. 2005; $73 \mathrm{~A}(2): 234-43$.

17. Baran ET, Mano JF, Reis RL. Starch-chitosan hydrogels prepared by reductive alkylation cross-linking. J Mater Sci: Mater Med. 2004;15(7):759-65.

18. Wool RP, Raghavan D, Wagner GC, Billieux S. Biodegradation dynamics of polymer-starch composites. J Appl Polym Sci. 2000; 77(8):1643-57.

19. Boesel LF, Azevedo HS, Reis RL. Incorporation of alpha-amylase enzyme and a bioactive filler into hydrophilic, partially degradable, and bioactive cements (HDBCs) as a new approach to tailor simultaneously their degradation and bioactive behavior. Biomacromolecules. 2006;7(9):2600-9.

20. Caldwell CG, Wurzburg, Otto B (1954) Unsaturated starch compounds and insoluble derivatives thereof. Inventor NAT STARCH PRODUCTS INC, assignee

21. International Standard ISO 5833. Implants for surgery-acrylic resins cements 1992.
22. Holm J, Lundquist I, Bjorck I, Eliasson AC, Asp NG. Degree of starch gelatinization, digestion rate of starch in vitro, and metabolic response in rats. Am J Clin Nutr. 1988;47(6):1010-6.

23. Di Paola RD, Asis R, Aldao MAJ. Evaluation of the degree of starch gelatinization by a new enzymatic method. Starch. 2003; 55(9):403-9.

24. Chinachoti P, Steinberg MP, Villota R. A Model for quantitating energy and degree of starch gelatinization based on water, sugar and salt contents. J Food Sci. 1990;55(2):543-6.

25. van der Burgt YEM, Bergsma J, Bleeker IP, Mijland PJHC, van der Kerk, van Hoof A, Kamerling JP, Vliegenthart JFG. Distribution of methyl substituents over crystalline and amorphous domains in methylated starches. Carbohydr Res 1999;320(1-2):100-7

26. Hebeish A, El-Alfy E, Bayazeed A. Synthesis of vinyl polymerstarch composites to serve as size base materials. Starch. 1988; 40(5):191-6.

27. Tomasik P, Schilling CH, Derek H. Chemical Modification of Starch. Advances in carbohydrate chemistry and biochemistry, Academic Press; 2004; 59:175-403.

28. Castellano I, Gurruchaga M, Goñi I. The influence of drying method on the physical properties of some graft copolymers for drug delivery systems. Carbohydr Polym. 1997;34(1-2):83-9.

29. Araújo PHH, Sayer C, Giudici R, Poço JGR. Techniques for reducing residual monomer content in polymers: a review. Polym Eng Sci. 2002;42(7):1442-68.

30. Hubbell JA. Biomaterials in tissue engineering. Bio/Technol. 1995;13(6):565-76. 